\title{
Actitudes lingüísticas sobre el uso de la lengua CH'ol en niños de tres comunidades indígenas de Tacotalpa, Tabasco
}

\section{Linguistic attitudes about the use of the $\mathrm{CH}$ 'ol language in children from three indigenous communities of Tacotalpa, Tabasco}

\author{
REYES-CRUZ, Emma†*, DE LOS SANTOS-RUIZ, Cynthia Paola y GUTIERREZ-CRUZ, Alberto \\ Mariano
}

Universidad Intercultural del Estado de Tabasco.

ID 1 $1^{\mathrm{er}}$ Autor: Emma, Reyes-Cruz / ORC ID: 0000-0002-3769-0629, CVU CONACYT ID: 953203

ID $1^{\mathrm{er}}$ Coautor: Cynthia Paola, De Los Santos-Ruiz / ORC ID: 0000-0003-2743-9200, CVU CONACYT ID: 1015872

ID $2^{\text {do }}$ Coautor: Alberto Mariano, Gutierrez-Cruz / ORC ID: 0000-0001-9799-6219, CVU CONACYT ID: 596534

DOI: $10.35429 / J P D L .2019 .17 .5 .17 .23$

Recibido 10 de Septiembre, 2019; Aceptado 30 Diciembre, 2019

\section{Resumen}

Esta investigación tuvo como objetivo identificar las actitudes lingüísticas sobre el uso de la lengua $\mathrm{CH}$ 'ol que tienen los niños de las comunidades de Cuviac, San Manuel y Puxcatán, Tacotalpa, Tabasco. La metodología fue cualitativa, el trabajo de campo se realizó con base en el diálogo de saberes (Left, 2014). Para ello, se trabajó con 3 niños de entre ocho y nueve años de las comunidades seleccionadas. Previo a este trabajo se llevaron a cabo 3 talleres -uno en cada comunidad- para socializar con los niños en las escuelas primarias, lo que permitó la selección de informantes para la entrevista. La selección de las comunidades fue con base en tres elementos: 1) que en la comunidad se hablara la lengua $\mathrm{CH}^{\prime}$ ol, 2) que pertenezca al municipio de Tacotalpa y 3) que al menos en dos comunidades hubiera escuelas primarias bilingües. La selección de los niños fue con base en: 1) haber demostrado durante los talleres dominio del vocabulario $\mathrm{CH}^{\prime}$ ol y 2) disponibilidad para realizar la entrevista.

Actitudes lingüísticas, lengua CH'ol, Espacios de uso de la lengua

\begin{abstract}
This research was aimed at identifying those linguistic activities about the use of $\mathrm{CH}$ 'ol language that children from communities of Cuviac, San Manuel and Puxcatan, Tacotalpa, Tabasco have. The methodology was qualitative, the field work was carried out based on the knowledge dialogue (Left, 2014). We worked with three children about eight and nine years old from the selected communities. Before this, 3 workshops were carried out - one in each community - to socialize with children in elementary schools. This allowed us the selection of informants to interview. Communities selection was based on three elements: 1) $\mathrm{CH}$ 'ol language as the spoken one in the community, 2) location at the municipality of Tacotalpa and 3) that at least two communities had bilingual elementary schools. Children selection was based on: 1) those ones who prove competence about $\mathrm{CH}$ 'ol vocabulary during the workshops and 2) availability to participate in the interview.
\end{abstract}

Linguistic attitudes, CH'ol language, Language use spaces

Citación: REYES-CRUZ, Emma, DE LOS SANTOS-RUIZ, Cynthia Paola, GUTIERREZ-CRUZ, Alberto Mariano. Actitudes lingüísticas sobre el uso de la lengua $\mathrm{CH}$ ’ol en niños de tres comunidades indígenas de Tacotalpa, Tabasco. Revista de Filosofía y Cotidianidad. 2019, 5-17: 17-23

\footnotetext{
* Correspondencia del Autor (emmareyesc@ hotmail.com)

$\dagger$ Investigador contribuyendo como primer autor.
} 


\section{Introducción}

El estudio de las lenguas originarias es necesario tanto para salvaguardar y estudiar la estructura de estas lenguas como para concientizar y empoderar la cultura de una población que durante siglos ha sido segregada: los pueblos indígenas y sus herederos. El texto que aquí se presenta es resultado de la investigación, pero también de la docencia y la vinculación por parte de docentes de la Universidad Intercultural del Estado de Tabasco, así como de niños, profesores y padres de familia de tres comunidades indígenas del municipio de Tacotalpa, Tabasco.

La actitud lingüística es un tema que se relaciona con la conducta y el pensamiento. Ésta puede ser positiva o negativa en términos de este mismo proceso. El tema de las actitudes lingüísticas ha sido estudiado por diferentes investigadores. Fishbein y Ajzen (1975) basan su aportación teórica sobre este tema en la teoría de la acción razonada que señala cómo la intención conductual próxima denota la conducta real del individuo y da como resultado la actitud y la norma subjetiva que viven los sujetos hablantes de la lengua.

En ese sentido, Janés (2006) refiere las investigaciones de Lambert y Tucker (1972), Genesee, Lambert y Holobow (1986), Baker (1992), Fernández (2001), Álvarez et al. (2001), Huguet (2001), González y Huguet (2002), Lasagabaster (2003) y Huguet, (2005) las cuales han sido eje fundamental en las actitudes hacía la lengua. Uno de los aportes sobre el estudio de las actitudes lingüísticas ha sido ayudar a entender mejor qué factores, elementos e incidencia hay respecto de las lenguas en la educación bilingüe (Sánchez y Sánchez, 1992). Dos aproximaciones teóricas a este tema de estudio se relaciona con la perspectiva conductiva y la perspectiva mentalista (Janés, 2006). La primera está enfocada en la actitud que muestra el sujeto, es decir las interacciones que tiene la lengua en sus diferentes usos (Bierbach, 1988; Appel y Muysken, 1986). Sin embargo, esta perspectiva ignora la posibilidad de predecir una conducta diferente. A diferencia, la perspectiva mentalista menciona que las actitudes son consideradas como un estado mental o interior, que sus respuestas están condicionadas en relación con las formas de comportamiento (Appel y Muysken, 1986; Bierbach, 1988).
De esta manera, las actitudes lingüísticas condicionan la forma de pensar de los hablantes, principalmente ante situaciones como la migración, la modernidad y las relaciones sociales con otros sujetos.

Los aportes de Castillo (2006) refieren que las actitudes se presentan de manera individual, social, comunitaria y étnica. En el estudio de las actitudes lingüísticas se reconocen tres componentes: el cognoscitivo, el afectivo y el conductual (Janés, 2006). Estos tres componentes que señala la autora se basan en la propuesta de Castillo (2006) sobre los Modelos de las actitudes Linguíísticas.

La lengua es resultado de la interacción social. En ella confluyen diversos actores que se interrelacionan no sólo a través del habla sino de sus subjetividades, su historia local, las políticas de Estado y las relaciones interculturales.

Por lo tanto, esta investigación tuvo como objetivo identificar las actitudes linguísticas sobre el uso de la lengua $\mathrm{CH}$ 'ol que tienen los niños de las comunidades de Cuviac, San Manuel y Puxcatán, Tacotalpa, Tabasco.

\section{Metodología}

Esta investigación se realizó con base en el enfoque cualitativo a través del diálogo de saberes (Left, 2014) sustentado en un acercamiento holístico al proceso histórico del sujeto. Para ello, se trabajó con 3 niños de entre ocho y nueve años de las comunidades de Puxcatán, San Manuel y Cuviac, de Tacotalpa, Tabasco. Las tres comunidades son hablantes de la lengua $\mathrm{CH}$ 'ol.

Previo a este trabajo se llevaron a cabo 3 talleres -uno en cada comunidad- para socializar con los niños en las escuelas primarias. Durante los talleres se implementaron actividades lúdicas que permitió a los investigadores reconocer el espacio comunitario, la dinámica de los niños y encontrar las estrategias de selección para las entrevistas.

La selección de las comunidades fue con base en tres elementos: 1) que en la comunidad se hablara la lengua $\mathrm{CH}$ 'ol, 2) que pertenezca al municipio de Tacotalpa y 3 ) que al menos en dos comunidades hubiera escuelas primarias bilingües. 
La selección de los niños fue con base en: 1) haber demostrado durante los talleres dominio del vocabulario Ch'ol y 2) disponibilidad para realizar la entrevista.

Las entrevistas se aplicaron a tres niños, uno por comunidad; así como a madres o padres de estos niños. Las entrevistas se llevaron a cabo en las escuelas y otras más en los hogares de los niños.

\section{Resultado}

La información presentada se organiza en tres apartados generales, el primero tiene que ver con los hablantes, el uso de la lengua y el contexto sociocultural de la misma; el segundo con la actitud lingüística de los niños y el tercero con la actitud linguiística de los padres para enseñar el $\mathrm{CH}$ 'ol a sus hijos, esto último se presenta como un hallazgo ya que no estuvo considerado dentro del objetivo; no obstante durante el análisis se visualizó la importancia de los padres ante el uso de la lengua.

\section{El contexto sociocultural de los niños hablantes de la lengua CH'ol en Puxcatán, San Manuel y Cuviac Tacotalpa, Tabasco}

Las actitudes linguiísticas deben ser estudiadas de acuerdo con los hablantes y el contexto sociocultural de la lengua (Castillo, 2006). Éste se construye a partir de la colectividad. Las tres comunidades choles en mención son resultado de un proceso migratorio que inició a finales del siglo XIX y que no ha concluido. En el caso de Puxcatán, y de muchas comunidades del municipio de Tacotalpa, hay registros de vestigios arqueológicos de la cultura zoque (Terreros, 2006). Según la historia oral, la presencia de los choles de Chiapas que llegaron a Puxcatán y Cuviac data hacia finales del siglo XIX y durante todo el siglo XX.

Puxcatán tiene una población de 1, 288 habitantes de los cuales 725 hablan una lengua indígena. Mientras que Cuviac tiene una población de 376 habitantes de los cuales 284 hablan una lengua indígena (INEGI, 2010). Es decir, la lengua se ha desplazado entre un $44 \%$ y $25 \%$, aproximadamente. La comunidad de San Manuel también es resultado de un proceso migratorio de personas provenientes de Chiapas que hacia los años 80 llegaron en busca de tierras; aquí, la totalidad de la población es hablante de la lengua CH'ol.
Los casi 70 años de diferencia entre la fundación de las comunidades de Puxcatán y Cuviac con San Manuel dan testimonio de un proceso de pérdida de la lengua relacionado con la migración laboral, las políticas educativas, las relaciones interculturales con otras comunidades de Tabasco que no son hablantes del CH'ol y el cambio de identidad al que han sido sometidos de manera pacífica los choles de Tabasco. Durante el trabajo de campo se observó que son los abuelos los principales promotores del uso y práctica de los conocimientos tradicionales dentro de la comunidad.

Durante la investigación, se encontró que los niños choles solo se comunican en esta lengua con familiares o personas conocidas, por lo que existe desplazamiento de la lengua $\mathrm{CH}^{\prime}$ ol por el español. Este último refiere a los casos de los niños de Puxcatán y Cuviac quienes están dejando de aprender la lengua originaria. Es notorio observar que la lengua $\mathrm{CH}$ 'ol ha entrado en desuso como resultado de un desplazamiento de la lengua y el incremento del bilingüismo. Situación que acontece con muchas otras lenguas de México (Terborg y Garcia, 2011).

El uso y proceso de desplazamiento de la lengua CH'ol da cuenta de las relaciones de poder a la que se enfrenta una lengua minoritaria como ésta. Los más de 100 años de historia de las comunidades de Puxcatán y Cuviac hablan de la resistencia a no perder la lengua pero dan paso a actitudes lingüísticas que no la favorecen como el desinterés, la falta de docentes bilingües en la educación primaria, secundaria y bachillerato; así como de otras actividades que empoderen a la lengua originaria.

\section{Actitudes lingüísticas de los niños hablantes de la lengua CH'ol en Puxcatán, San Manuel y Cuviac Tacotalpa, Tabasco}

El aprendizaje de la lengua CH'ol por parte de los niños es distinto para cada comunidad. Pero en las tres es notorio el interés que tienen los menores por aprender la lengua de sus ancestros. Para el caso de Puxcatán y Cuviac, la lengua materna de los niños entrevistados es el español, el CH'ol lo han ido adquiriendo principalmente por parte de los abuelos y de talleres de instituciones como el Consejo Nacional para el Desarrollo de los Pueblos Indígenas (CDI). 
En este sentido, la lengua sólo es referida en los hogares para enunciar palabras cortas como "frijol", "pozol", "tortilla", "perro" o "maíz". Pero no son capaces de entablar una conversación. Aunque es de reconocer que sí comprenden la lengua y pueden responder a ella sin ningún problema con afirmaciones o negaciones cortas.

En el caso de San Manuel, la lengua materna de los niños es el CH'ol y ellos comprenden y hablan fluidamente esta lengua, misma que es empleada en todos los espacios como la casa, la escuela, la milpa, las calles de la comunidad y tienditas.

A donde voy en la tienda, ahí hablo CH'ol y ahí toy [sic] aprendiendo, todo se dice en CH'ol, en todo, en Agua Escondida, Madrigal porque ahí hablan puro español. Ahí nada más hablo el CH'ol, en la iglesia hablo el CH'ol, en la tienda, en la escuela, en la casa, en ta' mi abuela [sic], en casa de mi tía, en la calle. (Diálogo con Luis Ángel, 12 años, comunidad de San Manuel).

Vargas (2016) en una investigación similar a ésta explica que "las relaciones interculturales y los procesos de homogenización cultural, [...] han generado una nueva reorganización hacia nuevas prácticas discursiva $\mathrm{y}$, han definido los usos de las lenguas en diversos contextos socioculturales" (Vargas, 2016, p. 33). Uno de los niños explica el interés que él tiene por seguir hablando su lengua. "Yo solo aprendí la lengua, les enseño la lengua a mis hermanitos más pequeños (kpäsbeñob kijts'nob) todo lo hablamos en la lengua (Pejtyel tyi lak ty’añ), (Diálogo con Jesús Jiménez, 10 años, comunidad de San Manuel, 2018.)

Por otro lado, Ángel Sánchez, niño de Puxcatán, menciona "mi mamá me enseñaba cuando estaba chico" y recuerda que así fue como le enseñaron: "me escribía en un papelito las palabras y yo las leía". En relación a esta respuesta, existe una actitud positiva para aprender y hablar la lengua $\mathrm{CH}$ 'ol. El aprendizaje de la lengua $\mathrm{CH}^{\prime} \mathrm{ol}$ se da en las siguientes condiciones: 1) Con mayor frecuencia, ya no se enseña CH'ol en las escuelas, 2) los niños muestran por sí mismos un interés por aprender la lengua, 3) los abuelos son los principales promotores de la lengua.
En ese mismo sentido, Alexis Martínez niño informante de la comunidad de Cuviac externa cómo empezó a usar la lengua: "mi abuelita me enseñó a hablar, cuando hablo con ella y le respondo se pone contenta, primero no le entendía y ahora ya, pero me siento más contento cuando lo hablo en mi comunidad" (Dialogo con niño, 12 años, comunidad de Cuviac, 2018).

El aprendizaje de una lengua originaria por parte de los niños puede ser con base en el interés, inquietud y curiosidad por la misma (Juárez, 2016). A diferencia del desinterés y discriminación hacia las lenguas indígenas de hace poco más de seis décadas, la actitud de los extranjeros -también llamados kaxlanes por los choles- en la comunidad ha cambiado.

Cuando me ven [las personas que vienen de otra comunidad o ciudad], quieren que yo les enseñe, y de hecho aquí llegaron varias personas y quiere que yo le enseñé CH'ol, cuando llego con mi abuela hablo $\mathrm{CH}$ 'ol, quiere que les enseñe y yo les enseño, cuando voy al arroyo, ahí les enseño el CH'ol, me dicen que cómo se llama el frijol en CH'ol, así se llama les digo, me dicen cómo se llama el puerco, le digo chityam. (Diálogo con Luis Ángel, niño de 10 años, comunidad de San Manuel, 2018)

Por el contrario, durante el trabajo de campo en la comunidad de Puxcatán, al llegar al salón de clases que fue el primer contacto con los niños, se les preguntó quiénes hablaban el CH'ol y pocos levantaron la mano. Pero cuando se preguntó por la traducción de palabras como "gallina", "pato", "vaca", etc. Ellos sabían la respuesta. En esta comunidad la escuela primaria no es bilingüe.

La escuela primaria de la comunidad de San Manuel es bilingüe, y por parte del docente hay una actitud positiva hacia la enseñanza y revitalización del CH'ol. Además de que él es hablante fluido de esta lengua, denota su orgullo por el CH'ol y practica todo el tiempo con sus estudiantes, aunado a ello, les enseña a hablar español. Por su parte, en la comunidad de Cuviac la institución es bilingüe, pero a través de los talles se pudo constatar que los docentes hacen escaso uso del $\mathrm{CH}^{\prime}$ ol en actividades académicas. 
Así pues, Alexis manifiesta que lo aprendido ha sido en casa, sin discriminación y abunda: "cuando voy a Tacotalpa no me da pena hablar CH'ol, hablo, pero a veces en la escuela sí. No me discriminan, pero no me siento en confianza" (Dialogo con Alexis Martínez, 12 años, comunidad de Cuviac, 2018).

Con base en las relaciones interculturales, se logró identificar que actualmente aquellas personas que no viven en la comunidad $\mathrm{CH}$ 'ol, siempre mantienen una actitud de interés por aprender la lengua, los niños se sienten motivados para enseñarla y no existe discriminación alguna en relación con la lengua.

\section{Actitudes lingüísticas de padres de familia de la lengua CH'ol en Puxcatán, San Manuel y Cuviac Tacotalpa, Tabasco}

La actitud que presentan los padres de familia es diversa. La historia de vida de generaciones que se enfrentaron al desempleo y son herederos de los peores años de discriminación lingüística en el estado de Tabasco, sobre todo en las comunidades de Puxcatán y Cuviac, les lleva a desafiar la cada vez más constante desaparición de hablantes de la lengua $\mathrm{CH}^{\prime}$ ol en su comunidad.

Rojas (2006) explica que para mantener la lengua y la cultura, es necesario que los hijos aprendan a leerla y escribirla para fortalecer la identidad. De esta manera se contribuye a que de manera intrínseca la lengua se mantenga con vida, se use y se revitalice. En este caso, es necesario reconocer que el CH'ol está pasando de ser una lengua oral a ser también una lengua escrita. Esto último le da esperanza de vida toda vez que sus hablantes viven un proceso de educación en diversas instituciones y su modo de comunicación no sólo es oral, sino también a través de la escritura.

El aprendizaje de la lengua CH'ol, en algunos casos, fue a través de sus padres "Mi papá y mi mamá me enseñaron a hablar la lengua" (Diálogo con José Méndez, 42 años, comunidad de San Manuel, 2018). Sin embargo, Guadalupe, madre de niño de Puxcatán explica que sabe "algunas palabras y lo aprendí porque en la casa que vivía se habla el CH'ol". Guadalupe no sabía CH'ol pero en casa de sus suegros se hablaba la lengua y ahí aprendió un poco.
En ambos casos, señalan que han vivido en un ambiente donde se practica la lengua $\mathrm{CH}$ 'ol y han enseñado a sus hijos aunque en circunstancias distintas.

La práctica cotidiana de la lengua, para el caso de la comunidad de Puxcatán, se ve fortalecida por las relaciones interétnicas con comunidades vecinas de Chiapas. "Como aquí vienen personas de Chiapas y no hablan español y pues le hablo en CH'ol. Poquito pero si le hablo en CH'ol". (Diálogo con Guadalupe, madre de niño de Puxcatán, 2018).

Esas conversaciones cortas con personas de Chiapas han sido practicadas por los niños quienes han aprendido a responder "Mach kom" o "Ma' añ", "no quiero" o "no hay", respectivamente. Lo anterior para responder cuando llegan a vender frutas y hortalizas de otras comunidades donde sólo se habla esta lengua. Es importante mencionar que este bilingüismo CH'ol - español ha sido desatendido y da cabida a un desplazamiento de la lengua originaria por el español, como lengua dominante y oficial.

En el caso de San Manuel, la actitud que muestran los padres hacia la lengua CH'ol ha sido para que a través de la lengua se continúe transmitiendo sus creencias y conocimientos; y permee la herencia cultural de familia en familia "para que conozcan los niños y comprendan. Les decimos que no aprendan castilla y no tiren nuestra lengua, de ahí ellos continúan hablándolo, también nos hablamos entre nosotros" (Diálogo con Jesús Jiménez, padre de niño de la comunidad San Manuel, 2018).

El fortalecimiento de la lengua es por una parte positiva entre ellos, mientras que la señora de Puxcatán, Guadalupe (2018) afirma “Algunas palabras [las aprende] porque él pregunta, oye cómo se dice eso en $\mathrm{CH}$ 'ol, pero de que yo vaya [a enseñarle] no. No lo sé decir". Por otro lado, José Méndez, padre del niño de la comunidad San Manuel refiere "Yo les mando a que aprendan a hablar la lengua para que no vaya a hablar mal, para que hable bien y con mucho respeto". La enseñanza de la lengua varía de una comunidad a otra, Guadalupe, madre de niño de Puxcatán comparte "[ $i$ enseñar a hablar la lengua CH'ol?] Yo le digo que respete a las personas mayores pero así normal. Casi que el CH'ol casi no, pues este ya casi acá no lo hablan" 
En Cuviac por su parte, Alexis aprendió a hablar CH'ol por la convivencia con su abuelita materna, y, al ser hijo de madre soltera tuvo que quedarse en la casa de ella mientras su madre trabajaba. Doña Josefa (abuela de Alexis) habla Ch'ol desde niña y usa ese idioma cuando la visitan sus familiares o amigos, también hace uso fluido del español. Ella menciona que no ha sufrido de discriminación.

Con los padre de Doña Josefa no cabía la discriminación, por el contrario manifiesta que siempre le enseñó a hacer uso de su lengua y a no sentirse menos solo por hablar CH'ol. Con esa idea le ha enseñado a su nieto "siempre le digo a Alexis, habla, siempre háblalo sin pena, y sí, él siempre quiere saber más" (Dialogo con Josefa, abuela del niño de la comunidad de Cuviac, 2018).

La forma de motivar a Alexis para seguir hablando y aprendiendo $\mathrm{CH}$ 'ol es mediante la inmersión en diálogos, "siempre lo motivo porque le hablo en $\mathrm{CH}$ 'ol, Alexis tráeme la leña [X-alexis ñuñs' añ tyej lak si'] ¿vas a comer? [¿mux a wuch'el?], y él siempre busca como responderme (Dialogo con Josefa, abuela del niño de la comunidad de Cuviac, 2018).

Es loable resaltar la importancia del contexto, ya que determina la enseñanza de la lengua: en el diálogo con el niño de Puxcatán, él es el interesado en rescatar la lengua y asiste a talleres por parte de la CDI, pregunta a los abuelos, a otros niños y practica su $\mathrm{CH}$ 'ol cuando le es posible. Lo mismo pasa con Alexis en Cuviac, el interés por su lengua (que no es su primera lengua), el apoyo de su abuela y sus compañeros de escuela le ha generado seguridad para fortalecer el uso del CH'ol. A diferencia del niño de la comunidad de San Manuel, donde está presente un interés más colectivo para seguir hablando el CH'ol.

El poco tiempo que dedican a hablar CH'ol la generación de adultos que hoy son padres de estos niños es resultado de la discriminación lingüística y de una educación escolar sólo en español. Hace falta empoderarlos a ellos con estrategias más holísticas y menos limitadas a talleres de aprendizaje de la lengua.

\section{Conclusiones}

Las actitudes lingüísticas de los niños de las comunidades de Puxcatán, Cuviac y San Manuel se han ido construyendo en función del constructo social. Sus actitudes lingüísticas responden a una forma de pensamiento que deriva de la cultura de cada comunidad y ésta a su vez de las relaciones interculturales que han construido con sus espacios de trabajo, la escuela y la familia.

Jóvenes que se casaron con un no hablante del CH'ol. Otros más que cada semana se van a trabajar a la ciudad o todos los días a la cabecera municipal y finalmente, la mayoría de ellos educados en una escuela donde se priorizó el español y se prohibió hablar la lengua CH'ol, dio como resultado que la mayoría de los niños ya no tenga como lengua materna el $\mathrm{CH}^{\prime}$ ol.

Es de reconocer la valiosa aportación lingüística y cultural de la comunidad de San Manuel. Sin embargo, la falta de empleo, el acceso a una educación sólo en español, la idea de Modernidad como la única oportunidad de mejorar sus condiciones de vida puede repetir el escenario de las otras dos comunidades.

Es urgente que a los esfuerzos institucionales también se sume la comunidad para enseñar la lengua. La literatura y los testimonios evidencian que los principales promotores de la lengua son los abuelos, y se desconoce de programas o acciones donde se les incluya en esta labor de preservación de las lenguas originarias.

\section{Referencias}

Appel R. y Muysken R. (1996) Bilingüismo y contacto de lenguas. España. Ariel.

Baker, C. (1992). Attitudes and Language. Clevedon: Multilingual Matters.

Bierbach, C. (1988). "Les actituds lingüístiques". En A. BAstardas \& J. Soler (eds.). Sociolingüística i llengua catalana. Barcelona: Empúries. 
Castillo, M. A. (2006). Mismo mexicano pero mismo idioma: identidades y actitudes lingüísticas en los maseualmej de los Cuetzalan. México. Universidad Nacional Autónoma de México - Instituto Nacional de Antropología e Historia. Recuperado de file:///D:/Downloads/9962-22072-1-PB.pdf

Fernández, A. (2001). Las actitudes lingüísticas en situaciones de contacto de lenguas: el caso de Galicia. Textos de Didáctica de la Lengua y de la Literatura, 26, 17-27.

Fishbein, M. y Ajzen, I. (1975). Belief, Attitude, Intention and Behaviour. Reading, Mass.: Addison-Wesley.

Instituto Nacional de Estadística y Geografía. (2010). Censo de Población y Vivienda. Recuperado de: https://www.inegi.org.mx/programas/ccpv/2010

Janés, J. (2006). Las actitudes hacia las lenguas y el aprendizaje lingüístico. Revista Interuniversitaria de Formación del Profesorado, (20), 2, 117-132. Recuperado de http://www.redalyc.org/pdf/274/27411341008.p df

Juárez, A. (2016). Actitudes lingüísticas de hablantes Náhuatl, factores de incidencia en la Educación Bilingüe en la comunidad de San Jerónimo, Amanalco, Texcoco. Edo. México. (Tesis de licenciatura inédita). Universidad Pedagógica Nacional, México.

Lastra, Y. (1992). Sociolingüística para hispanohablantes. Una introducción. México: El colegio de México.

Left, E. (2014). Diálogo de saberes, saberes locales y racionalidad ambiental en la construcción social de la sustentabilidad. Recuperado de https://redinterculturalidad.files.wordpress.com/ 2014/04/dic3a1logo-de-saberes-saberes-localesy-racionalidad-ambiental-en-la-

construccic3b3n-social-de-la-sustentabilidadenrique-leff.pdf

Rojas, C. (2006). Actitudes hacia la enseñanza de las lenguas indígenas. Recuperado de https://core.ac.uk/download/pdf/48864025.pdf.
Sánchez, M. P. y Sánchez, S. (1992). Psicología diferencial del aprendizaje de una segunda lengua. Valencia: Promolibro.

Terborg, R. y García, L. (2011). Muerte y vitalidad de las lenguas indígenas y las presiones sobre sus hablantes. México: Universidad Nacional Autónoma de México.

Terreros, E. (2006). Arqueología zoque de la región serrana tabasqueña. Estudios Mesoamericanos. (7) 29 - 43. Recuperado de http://www.iifilologicas.unam.mx/estmesoam/u ploads/Vol\%C3\%BAmenes/Volumen\%207/arq uelogia_zoque_eladio_terreros.pdf

Vargas García, I. (2016). Claroscuro en la revitalización lingüística de Hñähñú del Valle del Mesquital. (Tesis de maestría). Universidad Nacional Autónoma de México. Recuperado de http://www.linguapax.org/wpcontent/uploads/2015/03/Claroscuros_ItzelVarg as.pdf 